\title{
Oscillations in an active region filament: Observations and comparison with MHD waves
}

\begin{abstract}
S. Régnier, J. Solomon, and J. C. Vial
Institut d'Astrophysique Spatiale, Unité Mixte CNRS-Université Paris XI, bâtiment 121, 91405 Orsay Cedex, France

Received 13 October 2000 / Accepted 18 June 2001

Abstract. During the MEDOC Campaign 4, on October 1999, observations of a solar active region filament were carried out by the SUMER/SoHO spectrometer. A time sequence of this filament has been obtained with a duration of $7 \mathrm{~h} 30 \mathrm{~min}$ and with a temporal resolution of $30 \mathrm{~s}$. The Fourier analysis of the line-of-sight Doppler velocities measured in the $584.33 \AA \mathrm{HeI}$ line allows us to detect oscillations in several ranges of periodicities (short periods: less than 5 min, intermediate periods: 6-20 min, and long periods: greater than 40 min). From a theoretical point of view, we consider the possible modes of oscillations of an active region filament. Following Joarder \& Roberts (1993), we treat the filament as a plasma slab embedded in a uniform magnetic field inclined at an angle $\phi$ to the long axis of the slab. Solving the dispersion equations for Alfvén waves and magnetoacoustic waves, primary and secondary mode frequencies appear to be non-equidistant. For the comparison between the observed and calculated frequencies, we outline an identification method of the oscillation modes in the observed filament. This identification provides a diagnostic of the filament: the angle between the magnetic field and the long axis of the slab is estimated to be $18^{\circ}$, and the magnetic field strength $B(\mathrm{G})$ is proportional to the square root of the density $\rho_{\mathrm{o}}\left(\mathrm{cm}^{-3}\right)$ in the slab, $B \sim 2.9 \times 10^{-5} \sqrt{\rho_{\mathrm{o}}}$.
\end{abstract}

Key words. Sun: filaments - Sun: oscillations - MHD - waves

\section{Introduction}

The existence of oscillations in solar prominences and filaments has been known for several years (see reviews by Schmieder 1989; Vial 1998; Oliver 1999). Three categories of periods can be defined (Molowny-Horas et al. 1997): short ( $<5 \mathrm{~min}$ ), intermediate (6-20 min), long (40-90 min) periods. Short-period oscillations have been detected in prominences observed at the limb. For a quiet prominence, intensity fluctuations in time revealed oscillations in the range 3-6 min (Blanco et al. 1999). By measuring the Doppler shift of the $\mathrm{H} \alpha$ line, Wiehr et al. (1984), Balthasar et al. (1986) and more recently Thompson \& Schmieder (1991) found indications of line-of-sight velocity oscillations in the range 3-6 min. The $5 \mathrm{~min}$ period which was detected both in the intensity and velocity variations was interpreted as being due to the shaking of the supporting magnetic field by the chromospheric and photospheric oscillations (Balthasar et al. 1988). However, in filaments observed near the disk center these oscillations are hard to detect. Malherbe et al. $(1981,1987)$ found that there are no $\mathrm{H} \alpha$ oscillations in both quiescent and active region

Send offprint requests to: S. Régnier,

e-mail: stephane.regnier@ias.u-psud.fr filaments in the range $1-10 \mathrm{mHz}$. Oscillations in the range 3-15 min have been found in the $10830 \AA \mathrm{HeI}$ line (Yi et al. 1991; Molowny-Horas et al. 1998), in OIV and SiIV lines (Bocchialini et al. 2001). Concerning long periods, line-of-sight velocity oscillations were detected in $\mathrm{H} \alpha$ in the range 50-80 min (Wiehr et al. 1984; Bashkirtsev et al. 1983; Bashkirtsev et al. 1984), and recently in the $\mathrm{HeI}$ line at $584.33 \AA$ with a period of about $2 \mathrm{~h} 30 \mathrm{~min}$ with the $\mathrm{CDS} / \mathrm{SoHO}$ spectrometer (Régnier et al. 1999).

On the theoretical side, Galindo-Trejo (1987) has studied the stability of different models of quiescent prominences. In particular, he found that for a Kippenhahn-Schlüter (1957) model, the prominence (defined as a slab perpendicular to the solar surface) is stable and oscillates perpendicularly to its plane with a typical period of $16 \mathrm{~min}$. Also, Roberts (1992) has compared long-period oscillations with three simple models. Joarder \& Roberts (1992a,b, 1993) have set up a model of prominence oscillations in which the slab is embedded in a magnetic field in the absence of gravity, and the calculated frequencies of Alfvén and magnetoacoustic waves are compared successfully with observations of 3-6 min oscillations (e.g. Balthasar et al. 1988) and around 16 min oscillations (Yi et al. 1991). Oliver et al. (1992) have studied 

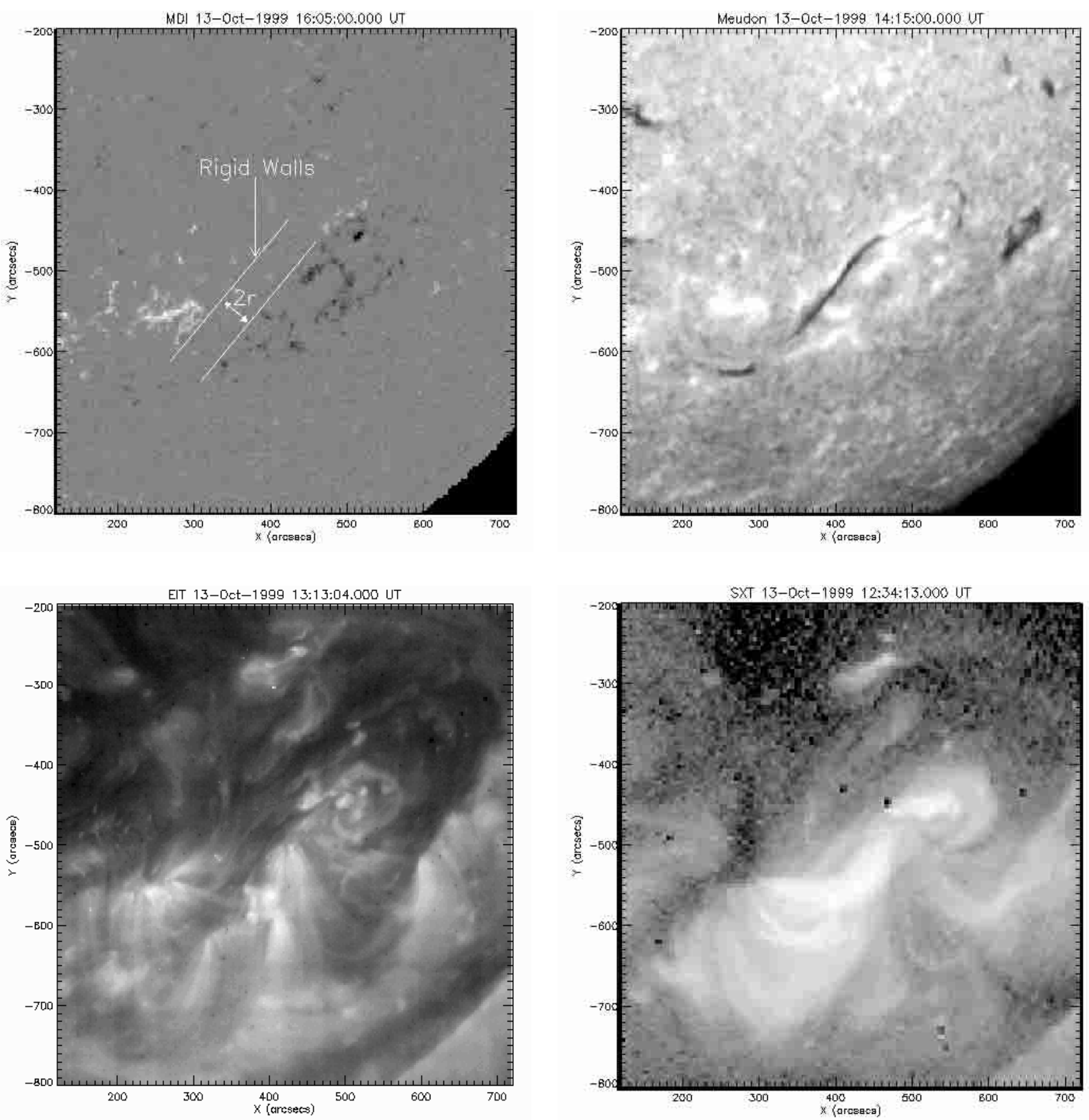

Fig. 1. Overview of AR 8725 on October 13, 1999: MDI magnetogram at 16:05 UT (top left - see Sect. 3.2 for the definition of the rigid walls), $\mathrm{H} \alpha$ image from Meudon observatory at 14:15 UT (top right - the filament is clearly visible as a dark feature at the center of the image). EIT image in the FeXII line at $195 \AA$ at 13:13 UT (bottom left), soft X-rays image from SXT/Yohkoh at 12:34 UT (bottom right).

the propagation of magneto-acoustic-gravity waves using a Kippenhahn-Schlüter model, and suggest that the short periods detected in prominences could be due to magneto-acoustic-gravity slow modes. More recently, the Joarder et al. (1997) model considers that the prominence consists of a large number of fibrils giving the observed sheet-like appearance of prominences. For a zero- $\beta$ plasma (without gas pressure), the authors conclude that the modes named fast kink modes produce the observed short periods $(2-15 \mathrm{~min})$. In the present paper, we interpret the observed oscillations in an active region filament in terms of magnetohydrodynamic (MHD) waves neglecting gravity, in order to provide a diagnostic of the filament.

In Sect. 2, we describe observations from the SUMER spectrometer and we perform a Fourier analysis of the temporal signal to obtain period ranges for the oscillations. In Sect. 3, the Joarder \& Roberts (1992a,b, 1993) model is used to obtain the dispersion relations and to find 


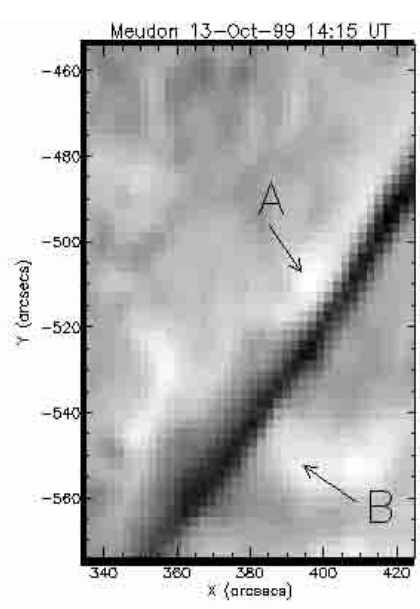

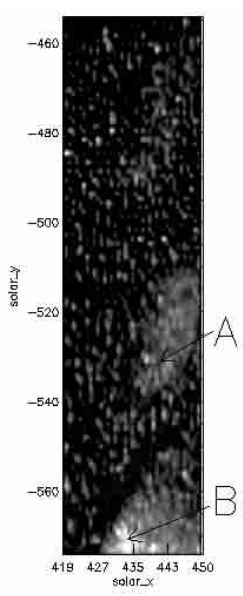

Fig. 2. Left: $\mathrm{H} \alpha$ image (see Fig. 1 top right; October 13 at 14:15 UT) focused on the filament. Two bright features $\mathrm{A}$ and $\mathrm{B}$ are defined on each side of the filament. Right: context raster obtained by SUMER in the HeI line at $584.33 \AA$ (October 13 at 22:11 UT). The dark feature between the two bright features (i.e., between $-565^{\prime \prime}$ and $-545^{\prime \prime}$ ) characterizes the filament.

the primary and secondary Alfvén and magnetoacoustic modes. In Sect. 4, we present an identification method of the observed filament oscillations in terms of Alfvén and magnetoacoustic waves. From this identification, we deduce a diagnostic of the filament. Conclusions are drawn in Sect. 5 .

\section{Observations with the SUMER/SoHO spectrometer}

A global description of the active region NOAA 8725 (AR 8725) observed on October 13-14, 1999 is provided (Fig. 1) by a magnetogram (MDI/SoHO) showing the distribution of the longitudinal magnetic field strength, an $\mathrm{H} \alpha$ image (Meudon observatory) in which the filament appears as a dark feature, a FeXII image at $195 \AA$ (EIT/SoHO) showing coronal loops and a filament channel, and a soft X-ray image (SXT/Yohkoh) in which the high temperature loops seem to have an S-shaped structure which is on top of the filament.

\subsection{Data set}

SUMER (Solar Ultraviolet Measurements of Emitted Radiation) is a normal incidence spectrograph on board SoHO (Wilhelm et al. 1995). During MEDOC Campaign 4, the sequence of SUMER observations discussed here was obtained from October 13 at 22:20 UT to October 14 at 05:50 UT (i.e., a duration of $7 \mathrm{~h} 30 \mathrm{~min}$ ). Using the $0.3^{\prime \prime} \times 120^{\prime \prime}$ slit, we observed the HeI line at $584.33 \AA$ with compensation of solar rotation. The exposure time was $30 \mathrm{~s}$, and the spectral resolution $20 \mathrm{~m} \AA$ (second order).

A context raster (Fig. 2 right) was made in the HeI line at the beginning and at the end of the observations: $0.3^{\prime \prime} \times 120^{\prime \prime}$ slit, field of view of $30^{\prime \prime} \times 120^{\prime \prime}$ (40 steps

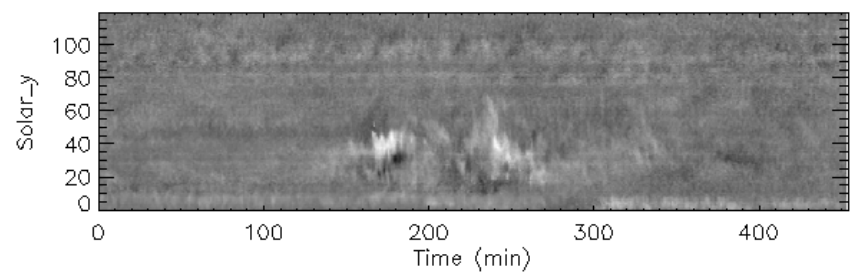

Fig. 3. Velocity time series derived from SUMER time series observations. The total duration is about $7 \mathrm{~h} 30 \mathrm{~min}$. White (black) corresponds to downwards/positive (upwards/negative) velocities.

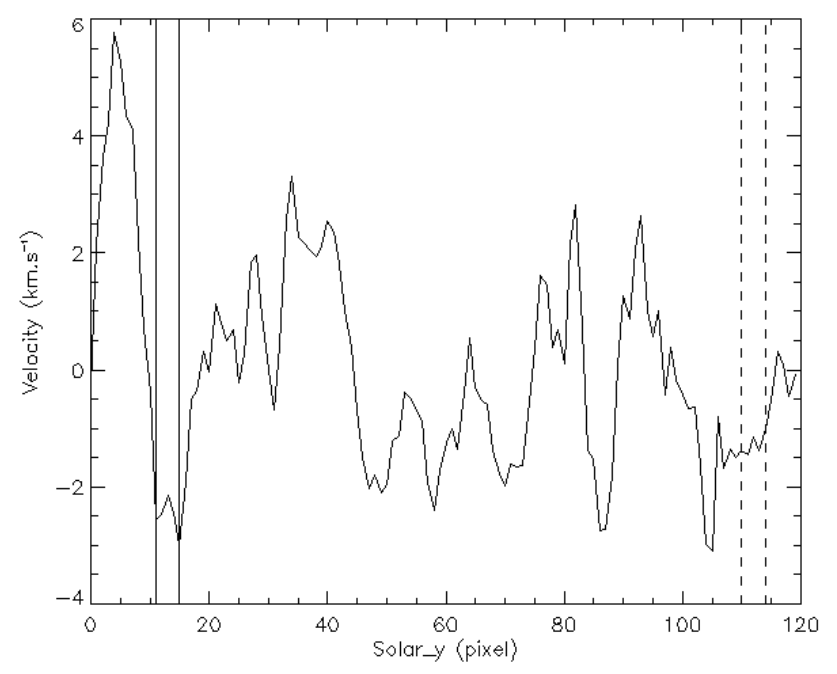

Fig. 4. Temporal average of the velocity time series. The position of the filament (resp. quiet region) is given by the two vertical solid lines (resp. dashed lines).

of $0.76^{\prime \prime}$ ), exposure time of 5 s. In Fig. 2 right, the filament corresponds to the dark feature between the two bright features A and B defined in Fig. 2 left. The location of the filament is estimated to be between the 5 th and the 25 th pixel along the slit (i.e. $-565^{\prime \prime}$ to $-545^{\prime \prime}$ ). The position of the slit for time series observations is in the middle of the $30^{\prime \prime} \times 120^{\prime \prime}$ field of view.

\subsection{Velocity time series}

The relative Doppler velocity $v$ is defined by $\frac{v}{c}=\frac{\Delta \lambda}{\lambda_{0}}$ where $c$ is the speed of light, $\lambda_{0}$ the wavelength at the maximum intensity of the mean profile obtained by the temporal and spatial average of the intensity time series, and $\Delta \lambda=\lambda-\lambda_{0}$ with $\lambda$ the Doppler displacement at a given time and at a given $(y)$ position along the slit. With this definition, we derive the velocity time series in which negative (positive) velocity corresponds to upwards (downwards) flows. The average velocity of the whole time series should be zero, estimating the contribution of the filament to be less than $5 \%$. Therefore in order to obtain the effective velocity time series (Fig. 3), we have 

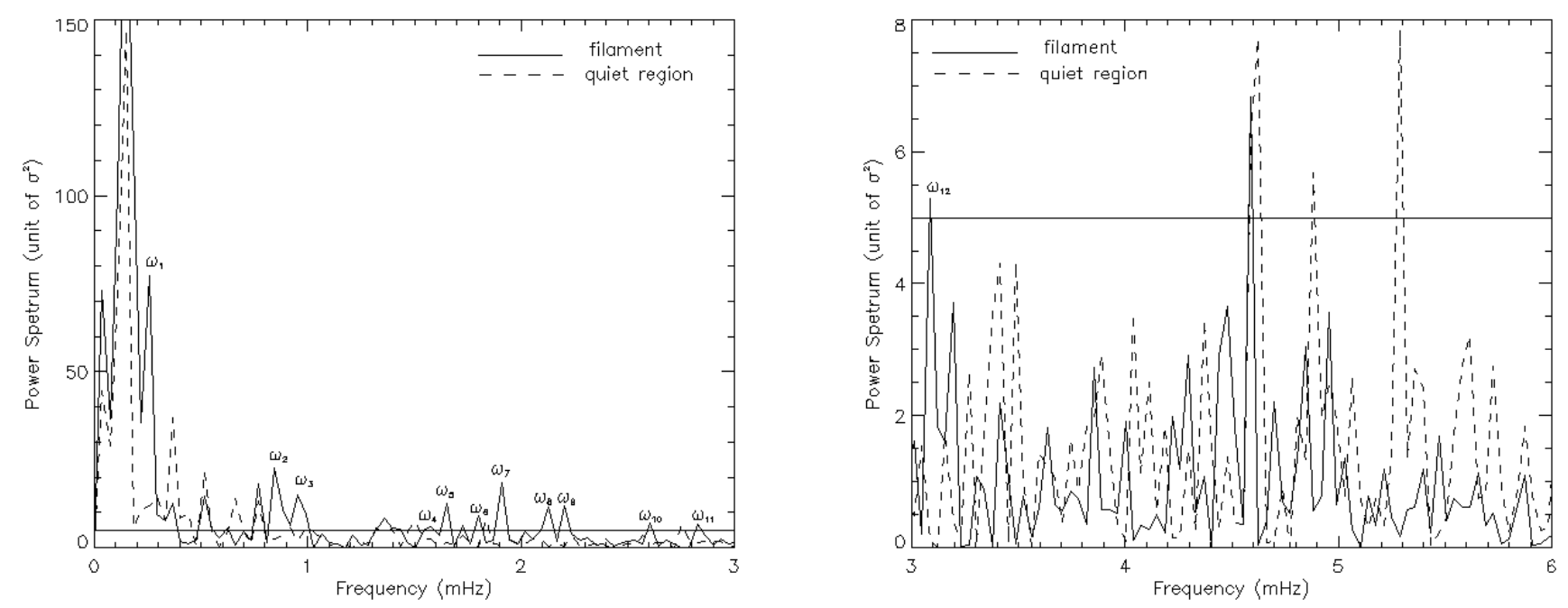

Fig. 5. Power spectrum calculated from the velocity time series of the filament (solid line) and for the quiet region (dashed line) for two ranges of frequencies (left: $<3 \mathrm{mHz}$, right: $3-6 \mathrm{mHz}$ ). The $99 \%$ confidence level is given by the horizontal solid line at $5 \sigma^{2}$. Each characteristic frequency of oscillation is annotated $\left(\omega_{n}\right)$.

subtracted the spatial and temporal averaged velocity of the raw velocities from the raw velocity time series.

To determine exactly the spatial $(y)$ location of the filament, we calculate the temporal average of the velocity time series (Fig. 4). The position of the filament can be defined precisely between the 11 th and the 15 th pixel along the slit (i.e. $-559^{\prime \prime}$ to $-555^{\prime \prime}$ ). In this area, the mean velocity is $-3 \mathrm{~km} \mathrm{~s}^{-1}$, implying that the filament rises in the solar atmosphere. For the sake of reference, we also choose a quiet area between the 110th and the 114th pixel (i.e. $-460^{\prime \prime}$ to $-456^{\prime \prime}$ ) which will provide the oscillation frequencies in the medium out of the filament.

\subsection{Fourier analysis}

For the effective velocity time series, we performed a Fourier analysis of the signal in the filament and in the quiet area. Because of the duration of the observations (7 $\mathrm{h} 30 \mathrm{~min})$ and the exposure time $(30 \mathrm{~s})$, the resulting frequencies range from $3.5 \times 10^{-2} \mathrm{mHz}$ to $15 \mathrm{mHz}$. The power spectrum deduced from the Fourier transform after an apodisation of the signal (Brault et al. 1971) yields $\mathcal{P}(\omega)=2|\widetilde{S}(t)|^{2}$ where $\omega$ is the frequency and $\widetilde{S}(t)$ is the Fourier transform of the time series.

In Fig. 5, the power spectrum in the filament (resp. in the quiet area) is normalized to its variance (the upper frequency is limited to $6 \mathrm{mHz}$ ). They exhibit a marked maximum for a frequency of about $0.15 \mathrm{mHz}$ which results from an instrumental thermal effect. Moreover for $0.3 \mathrm{mHz}<$ $\omega<0.8 \mathrm{mHz}$, there are common features between the filament and the quiet region which cannot be interpreted as characteristic oscillation frequencies in the filament. These features probably result from several instrumental effects such as pointing instabilities (Lemaire 2000). Note that no harmonic frequencies of the instrumental thermal effect are observed. In Fig. 5, the horizontal solid line defines
Table 1. The observed periods in the filament are deduced from the power spectra of Fig. 5. The intermediate (between 5 and $20 \mathrm{~min}$ in this case) and long (>40 min) periods exist.

\begin{tabular}{c|c|c}
\hline & \multicolumn{2}{|c}{ Observed Periods } \\
\hline $\begin{array}{c}\text { long } \\
\text { periods }\end{array}$ & $65 \mathrm{~min} 36 \mathrm{~s}$ & $\omega_{1}$ \\
\hline inter- & $19 \min 46 \mathrm{~s}$ & $\omega_{2}$ \\
mediate & $17 \min 28 \mathrm{~s}$ & $\omega_{3}$ \\
periods & $10 \min 36 \mathrm{~s}$ & $\omega_{4}$ \\
& $10 \min 07 \mathrm{~s}$ & $\omega_{5}$ \\
& $9 \min 17 \mathrm{~s}$ & $\omega_{6}$ \\
& $8 \min 46 \mathrm{~s}$ & $\omega_{7}$ \\
& $7 \min 50 \mathrm{~s}$ & $\omega_{8}$ \\
& $7 \min 34 \mathrm{~s}$ & $\omega_{9}$ \\
& $6 \min 24 \mathrm{~s}$ & $\omega_{10}$ \\
& $5 \min 54 \mathrm{~s}$ & $\omega_{11}$ \\
& $5 \min 24 \mathrm{~s}$ & $\omega_{12}$ \\
\hline short & not detected & \\
\hline
\end{tabular}

the $99 \%$ confidence level for a $\chi^{2}$ distribution with 2 degrees of freedom.

In Table 1, we list the characteristic oscillation frequencies in the filament. In particular, we find a period of oscillation larger than $40 \mathrm{~min}$. In this paper, we only focus on the existence of characteristic frequencies in the filament (the phases and the absolute values of the power are not investigated). Note that the $3 \mathrm{~min}$ chromospheric oscillations are observed in Fig. 5 right (frequencies close to $5 \mathrm{mHz}$ ). 


\subsection{Some features of the $584.33 \AA$ Hel line}

One may be surprised to be able to detect Doppler shifts in a line such as $584.33 \AA$. Let us recall that this line corresponds to the $1 \mathrm{~s}^{2}{ }^{1} \mathrm{~S}-1 \mathrm{~s} 2 \mathrm{p}{ }^{1} \mathrm{P}^{0}$ transition of parahelium. It has been measured in the solar chromosphere with different techniques (Dupree \& Reeves 1971; Dupree et al. 1973; Delaboudinière \& Crifo 1976; Cushman \& Rense 1978). The chromospheric profiles (Cushman \& Rense 1978) were rather broad and even possibly self-reversed (1976). This was a clear indication that the line is optically thick in the chromosphere. In prominences, no detailed measurement had been made until SoHO. A FWHM of about $14 \mathrm{~m} \AA$ was found in the quiet sun (Vial 2000) but there are clear indications that the line profile is narrower in prominences (Régnier et al. 2001) and certainly unreversed. All nonLTE models (Heasley et al. 1974) provide rather large line opacities in the range $10^{4}-10^{6}$ for prominences. Recent computations by Labrosse et al. (1999) provide similar results in the range $10^{4}-10^{5}$. These theoretical opacities are compatible with an unreversed profile (as measured with the SUMER/SoHO spectral resolution): actually, the low Doppler width (less than $15 \mathrm{~m} \AA$ if the microturbulence is lower than $5 \mathrm{~km} \mathrm{~s}^{-1}$ ) combined with an opacity of the order of $10^{5}$ leads to a transparent medium in the line wings beyond about 4 Doppler widths, i.e. $60 \mathrm{~m} \AA$. This means that the medium is essentially (but not totally) opaque in the line. Consequently, the information concerning the velocities associated with the measured line Doppler shifts should be considered as coming from a rather superficial layer of the prominence where the coherence of oscillations is retained.

\section{Filament model}

\subsection{Description of the model}

To examine the different modes of oscillations in a filament, we here consider the prominence model of Joarder \& Roberts (1993). In the frequency analysis defined in Sect. 2, the velocity time series is averaged along the slit ( $y$ spatial dimension), therefore we only study the global modes of oscillations in the filament. This fact justifies the use of the Joarder \& Roberts model in preference to the Joarder et al. (1997) model including the filament fine structures. The filament described as a simple slab is embedded in a skewed uniform magnetic field making an angle $\phi$ to the long axis of the slab (see Fig. 6). The effect of gravity and field line curvature are ignored. The slab is defined by a temperature $T_{\mathrm{o}}$, a density $\rho_{\mathrm{o}}$, a length $L$ in the $y$-direction, a height $H$ in the $z$-direction, and a width $2 a$ in the $x$-direction $(2 a \ll H, L)$. Its environment is defined by a temperature $T_{\mathrm{e}}$, and a density $\rho_{\mathrm{e}}$. The uniform magnetic field permeating the medium (the slab and its environment) is $\boldsymbol{B}=\left(B_{x}, B_{y}, 0\right)$ with $B_{x}=B_{y} \tan (\phi)$. In this case, the pressure balance between the slab and its environment implies that $\rho_{\mathrm{o}} T_{\mathrm{o}}=\rho_{\mathrm{e}} T_{\mathrm{e}}$. The magnetic field

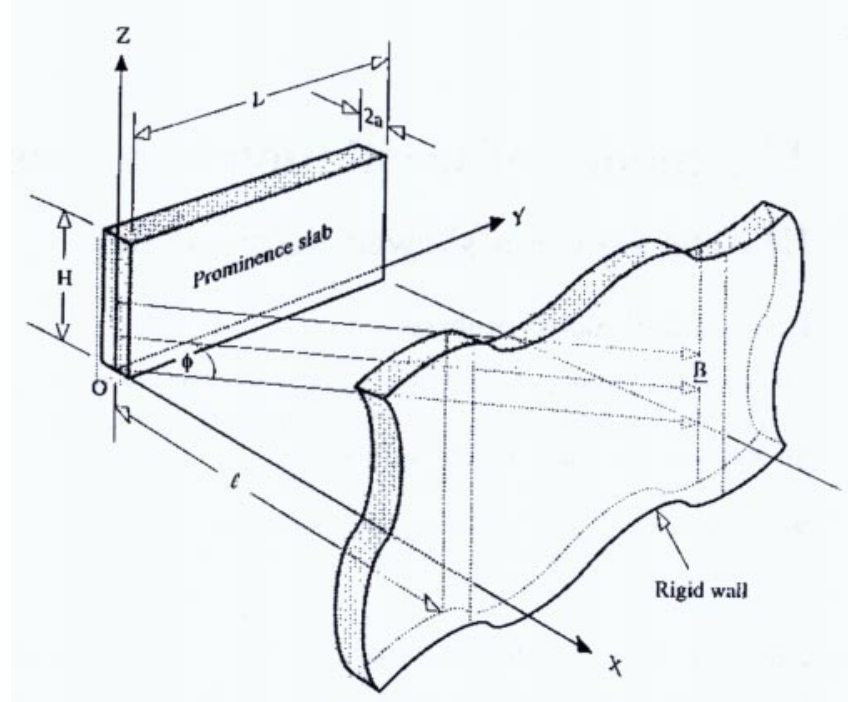

Fig. 6. A schematic diagram showing the filament as a dense plasma slab of width $2 a$ in the $x$-direction, length $L$ in the $y$-direction, and height $H$ in the $z$-direction. The slab is embedded in a magnetic field $\boldsymbol{B}$ lying in the $x y$-plane, making an angle $\phi$ to the long axis of the slab, and tying to rigid conducting walls at $x= \pm l$ (Joarder \& Roberts 1993).

lines are tied to rigid walls at $x= \pm l$ on either side of the slab to represent the photospheric line-tying effect.

The equations of ideal MHD (e.g. Priest 1982) are linearized about the slab equilibrium depicted in Fig. 6 (Joarder \& Roberts 1993), with the disturbance for an uniform unbounded medium assumed to be of the form:

$\delta \xi=\xi \exp \left(i \omega t-i k_{x} x-i k_{y} y-i k_{z} z\right)$

Here $\delta \xi$ is the disturbed quantity (gas pressure, velocity, or magnetic field), $\omega$ is the frequency of the disturbance, and $k_{x}, k_{y}, k_{z}$ are the wavenumbers in each direction. The perpendicular wavenumber is $k_{\perp}=\left(k_{y}^{2}+k_{z}^{2}\right)^{1 / 2}$. The characteristic speeds that enter in the description of modes of oscillations are the sound speed $c_{\mathrm{S}}$, the Alfvén speed $v_{\mathrm{A}}$ $\left(v_{\mathrm{A}}^{2}=v_{\mathrm{A} x}^{2}+v_{\mathrm{A} y}^{2}=B^{2} / \mu_{0} \rho\right.$ with $\left.B^{2}=B_{x}^{2}+B_{y}^{2}\right)$, the magnetoacoustic slow speed $c_{\mathrm{T}}=c_{\mathrm{s}} v_{\mathrm{A}} /\left(c_{\mathrm{s}}^{2}+v_{\mathrm{A}}^{2}\right)^{1 / 2}$, and the magnetoacoustic fast speed $c_{\mathrm{f}}=\left(c_{\mathrm{s}}^{2}+v_{\mathrm{A}}^{2}\right)^{1 / 2}$. These speeds carry the index "o" in the slab, and the index "e" in its environment.

In each medium and in the limit of extremely long wavelengths $\left(k_{\perp} l \ll 1\right.$; equivalently, $\left.k_{y}=k_{z}=0\right)$, three wavenumbers allow to describe the Alfvén modes and the magnetoacoustic modes: $\omega / v_{\mathrm{A} x}$ for Alfvén modes, $\omega / c^{+}$ for slow modes, and $\omega / c^{-}$for fast modes, where

$c^{ \pm}=\sqrt{2} v_{\mathrm{A} x} c_{\mathrm{s}}\left[c_{\mathrm{f}}^{2} \pm\left(c_{\mathrm{f}}^{4}-4 v_{\mathrm{A} x}^{2} c_{\mathrm{s}}^{2}\right)^{1 / 2}\right]^{-1 / 2}$.

The boundary conditions are given by the line-tying effect at $x= \pm l\left(v_{x}( \pm l)=v_{y}( \pm l)=v_{z}( \pm l)=0\right)$, and we also use the continuity conditions across $x= \pm a$ of the velocity $v_{x}$ and of the total pressure implying in this model the continuity of $\delta \boldsymbol{v}, \delta p, \delta B_{y}, \delta B_{z}$. In the linear system 
of equations resulting from boundary and continuity conditions, the Alfvén waves are decoupled from the magnetoacoustic waves. For Alfvén modes and magnetoacoustic modes, we consider symmetric modes (even Alfvén mode, slow and fast kink magnetoacoustic modes) and antisymmetric modes (odd Alfvén mode, slow and fast sausage magnetoacoustic modes). We obtain a dispersion relation for each of the six modes. The dispersion relations yield (see Joarder \& Roberts 1993):

$\tan \left(\frac{\omega a}{v_{\mathrm{A} x \mathrm{o}}}\right)-\sqrt{\frac{\rho_{\mathrm{e}}}{\rho_{\mathrm{o}}}} \cot \left(\frac{\omega(l-a)}{v_{\mathrm{A} x \mathrm{e}}}\right)=0$

for even Alfvén mode,

$\cot \left(\frac{\omega a}{v_{\mathrm{A} x \mathrm{o}}}\right)+\sqrt{\frac{\rho_{\mathrm{e}}}{\rho_{\mathrm{o}}}} \cot \left(\frac{\omega(l-a)}{v_{\mathrm{A} x \mathrm{e}}}\right)=0$

for odd Alfvén mode,

$\tan \left(\frac{\omega a}{c_{\mathrm{o}}^{ \pm}}\right)-\sqrt{\frac{\rho_{\mathrm{e}}}{\rho_{\mathrm{o}}}} \cot \left(\frac{\omega(l-a)}{c_{\mathrm{e}}^{ \pm}}\right)=0$

for slow (+) and fast (-) kink magnetoacoustic modes, and

$\cot \left(\frac{\omega a}{c_{\mathrm{o}}^{ \pm}}\right)+\sqrt{\frac{\rho_{\mathrm{e}}}{\rho_{\mathrm{o}}}} \cot \left(\frac{\omega(l-a)}{c_{\mathrm{e}}^{ \pm}}\right)=0$

for slow $(+)$ and fast $(-)$ sausage magnetoacoustic modes. For each dispersion relation, we define the primary frequency as the lowest frequency satisfying the dispersion relation, and the secondary frequencies as all the frequencies greater than the primary frequency. The primary and secondary frequencies for a single mode form a non-equidistant spectrum (see Table 2). Assuming that $a / l \ll 1$, the approximate solutions for primary frequencies are

$\omega \sim \frac{v_{\mathrm{o}}}{2 \pi(l a)^{1 / 2}}$

for even Alfvén mode $\left(v_{\mathrm{o}}=v_{\mathrm{A} x \mathrm{o}}\right)$ or kink magnetoacoustic modes $\left(v_{\mathrm{o}}=c_{\mathrm{O}}^{ \pm}\right)$in the slab, and

$\omega \sim \frac{v_{\mathrm{o}}}{4 a}$

for odd Alfvén mode $\left(v_{\mathrm{o}}=v_{\mathrm{A} x \mathrm{o}}\right)$ or sausage magnetoacoustic modes $\left(v_{\mathrm{o}}=c_{\mathrm{o}}^{ \pm}\right)$in the slab. The approximated primary and secondary frequencies are summarized in Table 2.

The ratio of the primary frequency for the even Alfvén mode (slow and fast kink modes respectively) by the primary frequency for the odd Alfvén mode (slow and fast sausage modes respectively) is given by:

$\eta=\frac{\omega_{\mathrm{eAm}}}{\omega_{\mathrm{oAm}}}=\frac{\omega_{\mathrm{skm}}}{\omega_{\mathrm{ssm}}}=\frac{\omega_{\mathrm{fkm}}}{\omega_{\mathrm{fsm}}}=\frac{2}{\pi}\left(\frac{a}{l}\right)^{1 / 2}$.

(eAm: even Alfvén mode, oAm: odd Alfvén mode, skm: slow kink mode, ssm: slow sausage mode, fkm: fast kink mode, fsm: fast sausage mode).

This ratio only depends on the dimensionless parameter $\left(\frac{a}{l}\right)$, and is independent of the density, the magnetic
Table 2. Primary and secondary approximated frequencies for even and odd Alfvén modes. Note that the secondary frequencies can be divided into the internal and external frequencies. For the kink modes (resp. sausage modes), $v_{\mathrm{A} x \mathrm{o}}$ and $v_{\mathrm{A} x \mathrm{e}}$ have to be replaced by $c_{\mathrm{o}}^{ \pm}$and $c_{\mathrm{e}}^{ \pm}$in the approximated frequencies of even Alfvén modes (resp. odd Alfvén modes).

\begin{tabular}{|c|c|c|}
\hline & primary frequency & secondary frequency \\
\hline \multirow[t]{2}{*}{$\begin{array}{l}\text { even Alfvén } \\
\text { mode }\end{array}$} & \multirow[t]{2}{*}{$\frac{v_{\mathrm{A} x \mathrm{O}}}{2 \pi(l a)^{1 / 2}}$} & $\begin{array}{c}\frac{n v_{\mathrm{A} x \mathrm{o}}}{2 a} \quad n=1,2, \ldots \\
\text { (internal mode) }\end{array}$ \\
\hline & & $\begin{array}{c}\frac{n v_{\mathrm{A} x \mathrm{e}}}{2(l-a)} \quad n=1,2, \ldots \\
(\text { external mode })\end{array}$ \\
\hline \multirow[t]{2}{*}{$\begin{array}{l}\text { odd Alfvén } \\
\text { mode }\end{array}$} & \multirow[t]{2}{*}{$\frac{v_{\mathrm{A} x \mathrm{O}}}{4 a}$} & $\begin{array}{c}\frac{(2 n+1) v_{\mathrm{A} x \mathrm{O}}}{4 a} \quad n=1,2, \ldots \\
\quad \text { (internal mode) }\end{array}$ \\
\hline & & $\begin{array}{c}\frac{n v_{\mathrm{A} x \mathrm{e}}}{2(l-a)} \quad n=1,2, \ldots \\
\quad \text { (external mode) }\end{array}$ \\
\hline
\end{tabular}

field strength and the angle $\phi$ between the magnetic field and the long axis of the slab. Therefore in principle, the identification of the different modes is made possible by the observational estimation of the parameters $a$ and $l$, as described below.

\subsection{Method to identify the existing modes}

We solve the dispersion relations using the parameters of AR 8725 described in Sect. 2. We estimate the width of the filament from the $\mathrm{H} \alpha$ image (Fig. 1 top right): $2 a \sim 8000 \mathrm{~km}$. The length $l \sim 63000 \mathrm{~km}$ is deduced from the MDI magnetogram: the rigid walls are defined as the two lines (see Fig. 1 top left) parallel to the filament and separated of $2 r$, and in a cylindrical hypothesis for the shape of the magnetic field lines the length $l$ is given by $\frac{\pi}{2} r$. We choose typical values for the temperature of the filament $T_{\mathrm{o}}=8000 \mathrm{~K}$ and of its environment $T_{\mathrm{e}}=10^{6} \mathrm{~K}$, for the density of the slab $\rho_{\mathrm{o}}=10^{12} \mathrm{~cm}^{-3}$ (Schmieder et al. 1985) implying a density of its environment (since $\rho_{\mathrm{o}} T_{\mathrm{o}}=\rho_{\mathrm{e}} T_{\mathrm{e}}$ ) of $8 \times 10^{9} \mathrm{~cm}^{-3}$, for the magnetic field strength $B=20 \mathrm{G}$, and for the angle between the magnetic field and the long axis of the slab $\phi=25^{\circ}$ (Leroy et al. 1984).

In Table 3, we summarize the primary and secondary periods higher than 3 min derived from a model with a density of $10^{12} \mathrm{~cm}^{-3}$, a magnetic field strength of $20 \mathrm{G}$, and an angle $\phi$ of $25^{\circ}$. For slow (kink and sausage) magnetoacoustic modes, a large number of secondary periods exists particularly in the range $3-6 \mathrm{~min}(2.5-5 \mathrm{mHz})$.

We examine the dependence of the calculated primary frequency on the angle $\phi$ (Fig. 7) with a magnetic field strength $B=20 \mathrm{G}$ and a density $\rho_{\mathrm{o}}=10^{12} \mathrm{~cm}^{-3}$. The fast (kink and sausage) magnetoacoustic mode frequencies are constant when the angle $\phi$ changes. For small angles, one retrieves the special case of purely longitudinal static magnetic field (Joarder \& Roberts 1992a). For angles close 
Table 3. Primary and secondary periods for a model with a density of $10^{12} \mathrm{~cm}^{-3}$, a magnetic field strength of $20 \mathrm{G}$, and an angle $\phi$ of $25^{\circ}$, for periods higher than 3 min.

\begin{tabular}{|c|c|c|}
\hline & Primary periods & Secondary periods \\
\hline Slow kink mode & $5 \mathrm{~h} 46 \mathrm{~min} 51 \mathrm{~s}$ & $\begin{array}{l}37 \min 48 \mathrm{~s}, 27 \min 28 \mathrm{~s}, 18 \min 25 \mathrm{~s}, 14 \min 02 \mathrm{~s}, 12 \min 10 \mathrm{~s}, \\
9 \min 30 \mathrm{~s}, 9 \min 00 \mathrm{~s}, 7 \min 24 \mathrm{~s}, 6 \min 56 \mathrm{~s}, 6 \min 08 \mathrm{~s}, \\
5 \min 35 \mathrm{~s}, 5 \min 14 \mathrm{~s}, 4 \min 41 \mathrm{~s}, 4 \min 33 \mathrm{~s}, 4 \min 06 \mathrm{~s}, \\
3 \min 58 \mathrm{~s}, 3 \min 41 \mathrm{~s}, 3 \min 29 \mathrm{~s}, 3 \min 20 \mathrm{~s}\end{array}$ \\
\hline Even Alfvén mode & $69 \min 30 \mathrm{~s}$ & $7 \min 34 \mathrm{~s}, 5 \min 30 \mathrm{~s}, 3 \min 41 \mathrm{~s}$ \\
\hline Slow sausage mode & $57 \min 22 \mathrm{~s}$ & $\begin{array}{l}35 \min 56 \mathrm{~s}, 19 \min 33 \mathrm{~s}, 17 \min 33 \mathrm{~s}, 12 \min 23 \mathrm{~s}, 11 \min 04 \mathrm{~s}, 9 \min 12 \mathrm{~s} \text {, } \\
7 \min 59 \mathrm{~s}, 7 \min 20 \mathrm{~s}, 6 \min 17 \mathrm{~s}, 6 \min 03 \mathrm{~s}, 5 \min 17 \mathrm{~s}, 5 \min 03 \mathrm{~s} \text {, } \\
4 \min 36 \mathrm{~s}, 4 \min 17 \mathrm{~s}, 4 \min 04 \mathrm{~s}, 3 \min 44 \mathrm{~s}, 3 \min 39 \mathrm{~s}, 3 \min 21 \mathrm{~s}\end{array}$ \\
\hline Fast kink mode & $28 \min 53 \mathrm{~s}$ & $<3 \mathrm{~min}$ \\
\hline Odd Alfvén mode & $11 \min 29 \mathrm{~s}$ & $7 \min 12 \mathrm{~s}, 3 \min 55 \mathrm{~s}, 3 \min 31 \mathrm{~s}$ \\
\hline Fast sausage mode & $4 \min 46 \mathrm{~s}$ & $<3 \min$ \\
\hline
\end{tabular}

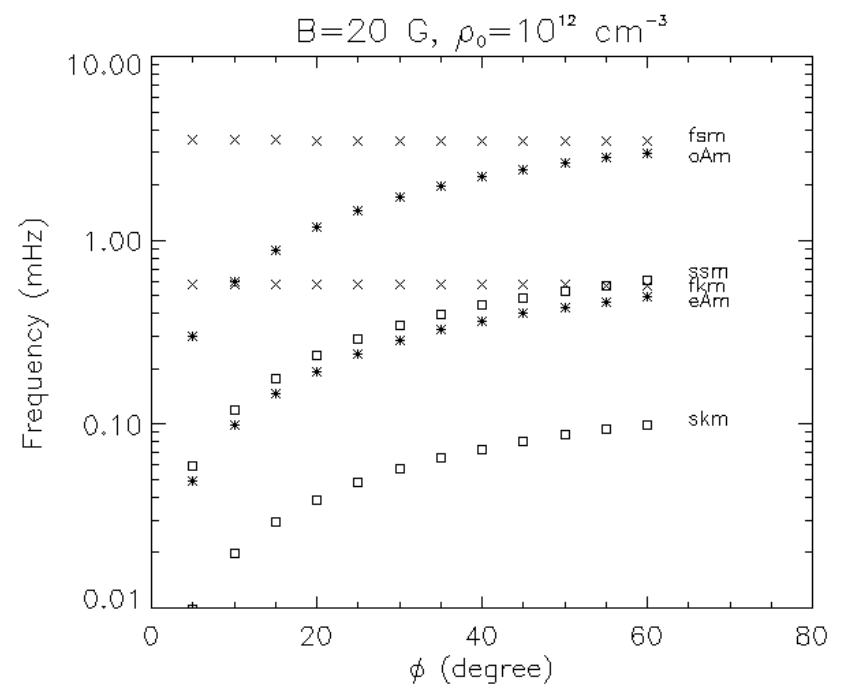

Fig. 7. Evolution of primary frequencies as a function of the angle $\phi$ for Alfvén and magnetoacoustic modes $(B=20 \mathrm{G}$, $\left.\rho_{\mathrm{o}}=10^{12} \mathrm{~cm}^{-3}\right)$. Fast mode frequencies $(\times)$ are constant, and Alfvén mode frequencies merge to fast mode frequencies for angles close to $90^{\circ} . *$ : odd Alfvén mode (oAm) and even Alfvén mode (eAm). $\square$ : slow sausage mode (ssm) and slow kink mode $(\mathrm{skm}) . \times$ : fast sausage mode (fsm) and fast kink mode $(\mathrm{fkm})$.

to $90^{\circ}$, the even (odd) Alfvén mode becomes similar to the fast kink (sausage) mode. As shown by Joarder \& Roberts (1992b), this last case of a purely transverse static magnetic field gives on one hand a fast kink (sausage) magnetoacoustic wave identical to the even (odd) Alfvén wave with the same speed $c_{\mathrm{f}}$ and on the other hand a slow (kink or sausage) magnetoacoustic wave with a characteristic speed $c_{\mathrm{T}}$. Note that for $\phi \sim 10^{\circ}$, the odd Alfvén mode and the fast kink mode have the same primary period of oscillations (around $30 \mathrm{~min}$ ).

In Fig. 8, we display the mode frequencies versus the magnetic field strength with a density $\rho_{\mathrm{o}}=10^{12} \mathrm{~cm}^{-3}$ and an angle $\phi=25^{\circ}$. The slow mode frequencies remain pratically constant when $B$ varies. For very low magnetic field strength, only slow modes exist with a characteristic speed equal to the sound speed. For a magnetic field

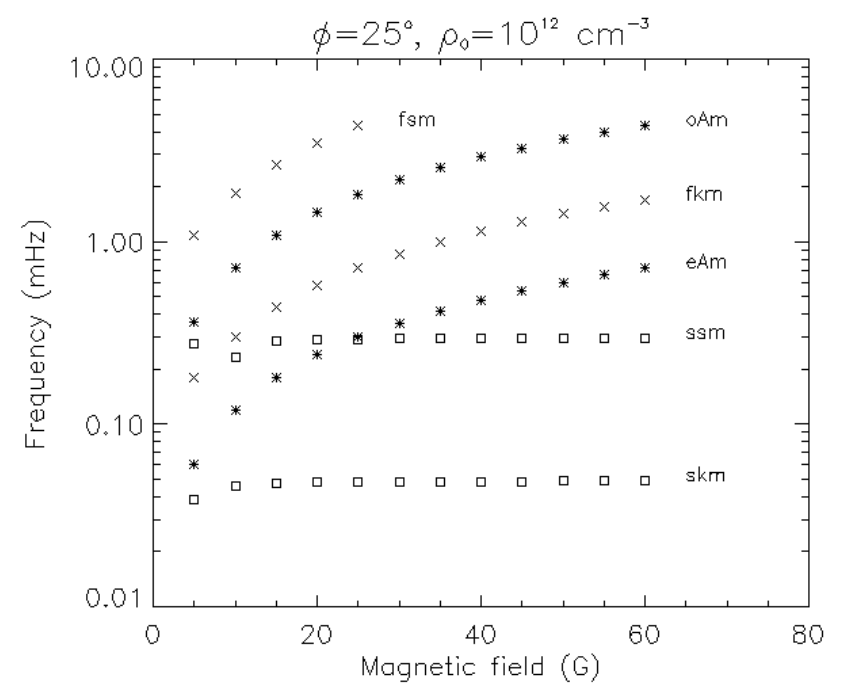

Fig. 8. Evolution of primary frequencies as a function of the magnetic field strength $B\left(\phi=25^{\circ}, \rho_{\mathrm{o}}=10^{12} \mathrm{~cm}^{-3}\right)$. Slow mode frequencies $(\square)$ are pratically constant.

strength of about $25 \mathrm{G}$, the even Alfvén mode intersects the slow sausage mode for a period of about 1 hour.

In Fig. 9, we plot the evolution of the primary frequencies as a function of the density for a magnetic field strength $B=20 \mathrm{G}$ and an angle $\phi=25^{\circ}$. The slow magnetoacoustic mode frequencies do not depend on the density. In any case (see Figs. 7-9), we observe that relative (logarithmic) distances in frequency of the odd Alfvén mode and the even Alfvén mode (fast sausage mode and fast kink mode, slow sausage mode and slow kink mode, respectively) do not vary in agreement with Eq. (8).

For a given value of the density in the slab $\rho_{\mathrm{o}}$, the even Alfvén mode and the slow sausage mode intersect for a magnetic field strength $B_{0}\left(\rho_{\mathrm{o}}\right)$ given by:

$B_{0}^{2}\left(\rho_{\mathrm{o}}\right)=\mu_{0} \rho_{\mathrm{o}} c_{\mathrm{so}}^{2}\left(\frac{1}{\eta^{2}}-1\right)$.

For a reasonable range of $\phi\left(10-90^{\circ}\right)$, we can order the Alfvén and magnetoacoustic mode frequencies (see Figs. 7-9, Table 3). If $B<B_{0}\left(\rho_{\mathrm{o}}\right)$ :

$\omega_{\mathrm{skm}}<\omega_{\mathrm{eAm}}<\omega_{\mathrm{ssm}}<\omega_{\mathrm{fkm}}<\omega_{\mathrm{oAm}}<\omega_{\mathrm{fsm}}$ 


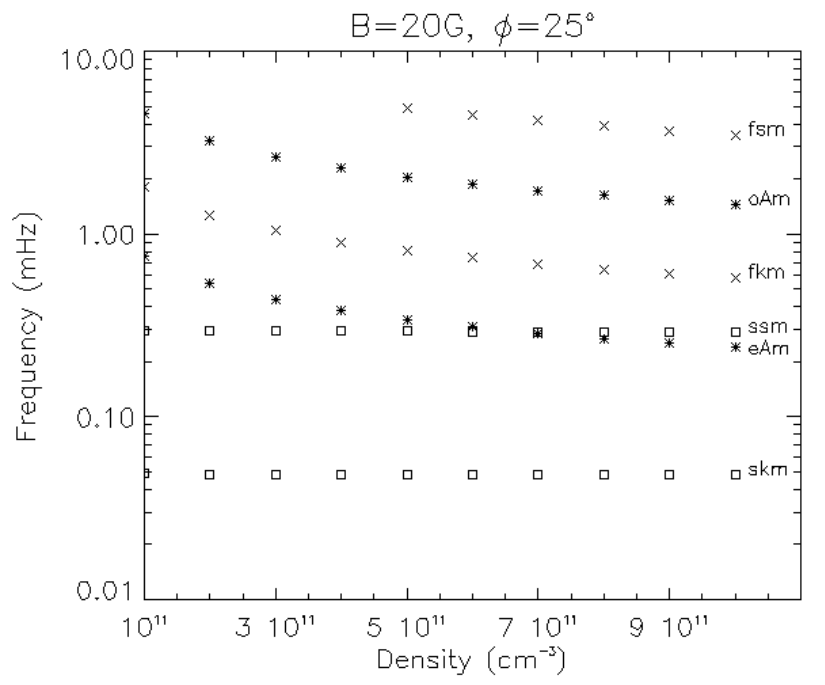

Fig. 9. Evolution of the primary frequencies as a function of the density in the slab, with $B=20 G, \phi=25^{\circ}$. Slow mode frequencies $(\square)$ do not depend on the density.

and if $B>B_{0}\left(\rho_{\mathrm{o}}\right)$ :

$\omega_{\mathrm{skm}}<\omega_{\mathrm{ssm}}<\omega_{\mathrm{eAm}}<\omega_{\mathrm{fkm}}<\omega_{\mathrm{oAm}}<\omega_{\mathrm{fsm}}$

(skm: slow kink mode, ssm: slow sausage mode, eAm: even Alfvén mode, oAm: odd Alfvén mode, fkm: fast kink mode, fsm: fast sausage mode).

Following the above parametric study, we outline a method to identify pratically the observed frequencies with respect to the calculated Alfvén or magnetoacoustic mode frequencies:

(i) for a given active region filament, we determine the dimensionless parameter $\eta$ (Eq. (8));

(ii) we calculate the ratios of the different observed frequencies that we compare with $\eta$;

(iii) we define the observational constraints to apply to the model: the lowest (largest) observed frequencies are limited by the total duration (exposure time) of the time series;

(iv) we eliminate the different ratios obtained in (ii) which do not satisfy the inequalities (10) or (11) and the conditions (iii).

\section{Application to the active region filament}

\subsection{Identification of the observed modes}

We apply the identification method to the observed frequencies found for the active region filament described in Sect. 2 (see Table 1):

(i) we calculate the dimensionless parameter $\eta$ with $2 a \sim$ $8000 \mathrm{~km}$ and $l \sim 63000 \mathrm{~km}: \eta=\mathbf{0 . 1 6}$. We consider the relative uncertainty to be $\sim 20 \%$ (given by the spatial resolution of the $\mathrm{H} \alpha$ image and the MDI magnetogram);
Table 4. The ratios of the different observed frequencies. The frequencies close to $\eta$ are indicated in bold characters. The ratios $\frac{\omega_{j>4}}{\omega_{i>j}}$ are larger than $\eta$.

\begin{tabular}{c|c|c|c|c}
\hline ratio & $\frac{\omega_{1}}{\omega_{i}}$ & $\frac{\omega_{2}}{\omega_{i}}$ & $\frac{\omega_{3}}{\omega_{i}}$ & $\frac{\omega_{4}}{\omega_{i}}$ \\
\hline$i=2$ & 0.305 & & & \\
$i=3$ & 0.269 & 0.884 & & \\
$i=4$ & $\mathbf{0 . 1 6 4}\left(\eta_{1}\right)$ & 0.536 & 0.607 & \\
$i=5$ & $\mathbf{0 . 1 5 6}\left(\eta_{2}\right)$ & 0.512 & 0.579 & 0.954 \\
$i=6$ & $\mathbf{0 . 1 4 3}\left(\eta_{3}\right)$ & 0.470 & 0.532 & 0.876 \\
$i=7$ & $\mathbf{0 . 1 3 5}\left(\eta_{4}\right)$ & 0.444 & 0.502 & 0.827 \\
$i=8$ & 0.121 & 0.396 & 0.449 & 0.739 \\
$i=9$ & 0.117 & 0.383 & 0.433 & 0.713 \\
$i=10$ & 0.099 & 0.324 & 0.367 & 0.604 \\
$i=11$ & 0.091 & 0.299 & 0.338 & 0.556 \\
$i=12$ & 0.083 & 0.273 & 0.309 & 0.510 \\
\hline
\end{tabular}

Table 5. Identification of the observed frequencies as primary frequencies of Alfvén and magnetoacoustic modes.

\begin{tabular}{c|c|c}
\hline & frequency $(\mathrm{mHz})$ & period \\
\hline slow kink mode & \multicolumn{2}{|c}{ not detected } \\
slow sausage mode & \multicolumn{2}{|c}{ not detected } \\
even Alfvén mode & $\omega_{1}=0.257$ & 65 min $36 \mathrm{~s}$ \\
fast kink mode & $\omega_{2}=0.843$ & $19 \min 46 \mathrm{~s}$ \\
odd Alfvén mode & $\omega_{5}=1.646$ & 10 min $07 \mathrm{~s}$ \\
fast sausage mode & \multicolumn{2}{|c}{ not detected } \\
\hline
\end{tabular}

(ii) using Table 1, we calculate the ratios of the different frequencies observed in the filament (see Table 4). The ratios close to $\eta$ (bold characters) are $\eta_{1}=\frac{\omega_{1}}{\omega_{4}}$, $\eta_{2}=\frac{\omega_{1}}{\omega_{5}}, \eta_{3}=\frac{\omega_{1}}{\omega_{6}}, \eta_{4}=\frac{\omega_{1}}{\omega_{7}}$;

(iii) since the total duration of the observations is $7 \mathrm{~h}$ $30 \min \left(3.5 \times 10^{-2} \mathrm{mHz}\right)$, the frequencies have to be greater than twice $3.5 \times 10^{-2} \mathrm{mHz}$ to be detected. Therefore, the slow kink mode is not detected in the reasonable ranges of $B$ and $\phi$ (Figs. 7-9);

(iv) we assume that the first observed frequency is a frequency which corresponds to a mode of oscillations. In Table 4, all ratios close to $\eta$ contain $\omega_{1}$. Therefore, the first observed mode is the even Alfvén mode: the slow sausage mode cannot be associated with a higher frequency, and consequently $\omega_{\mathrm{ssm}}<\omega_{\mathrm{eAm}}$, which is the case of the inequality (11). Then, we identify the observed frequencies $\omega_{2}$ to the fast kink mode $\omega_{\mathrm{fkm}}$ and $\omega_{5}$ to the odd Alfvén mode $\omega_{\mathrm{oAm}}$. In any case, we have chosen the largest peak.

We summarize these results in Table 5 . Note that the non-identified frequencies could be associated to one or several secondary frequencies of the MHD modes (see Table 3). 


\subsection{Diagnostic of the observed filament}

We use the results of Sect. 4.1 to obtain a diagnostic of the active region filament. The observed frequencies are linked to the approximate primary frequencies (see Eqs. (6) and (7)). The unknown parameters are the temperature in the slab $T_{\mathrm{o}}$, the angle $\phi$, the density in the slab $\rho_{\mathrm{o}}$ and the magnetic field strength $B$. The temperature $T_{\mathrm{o}}$ in the slab is a free parameter in this case. The equations to solve are:

$\omega_{\mathrm{eAm}}=\frac{v_{\mathrm{A} x \mathrm{o}}}{2 \pi(l a)^{1 / 2}}$,

$\omega_{\mathrm{fkm}}=\frac{c_{\mathrm{o}}^{-}}{2 \pi(l a)^{1 / 2}}$

where $v_{\mathrm{A} x \mathrm{O}}=\frac{B \sin \phi}{\sqrt{\mu_{0} \rho_{\mathrm{o}}}}$, and $c_{\mathrm{o}}^{-}=\sqrt{2} v_{\mathrm{A} x \mathrm{o}} c_{\mathrm{so}}\left[c_{\mathrm{fo}}^{2}-\left(c_{\mathrm{fo}}^{4}-\right.\right.$ $\left.\left.4 v_{\mathrm{A} x \mathrm{O}}^{2} c_{\mathrm{so}}^{2}\right)^{1 / 2}\right]^{-1 / 2}$.

Therefore, we obtain $v_{\mathrm{Ao}}$ and $\sin \phi$ as a function of $\omega_{\mathrm{eAm}}, \omega_{\mathrm{fkm}}$, and the temperature $T_{\mathrm{o}}$ :

$v_{\mathrm{Ao}}=\frac{1}{\omega_{\mathrm{fkm}}} \sqrt{c_{\mathrm{s}}^{2}\left(\omega_{\mathrm{eAm}}^{2}-\omega_{\mathrm{fkm}}^{2}\right)+4 \pi^{2} l a \omega_{\mathrm{fkm}}^{4}}$

$\sin \phi=\frac{2 \pi(l a)^{1 / 2} \omega_{\mathrm{eAm}} \omega_{\mathrm{fkm}}}{\left(c_{\mathrm{s}}^{2}\left(\omega_{\mathrm{eAm}}^{2}-\omega_{\mathrm{fkm}}^{2}\right)+4 \pi^{2} l a \omega_{\mathrm{fkm}}^{4}\right)^{1 / 2}}$.

For a temperature $T_{\mathrm{o}}=8000 \mathrm{~K}$ (e.g. Vial 1998), we estimate the angle $\phi$ :

$\phi=18^{\circ} \pm 2.5^{\circ}$,

and we obtain a relation between $B$ and $\rho_{\mathrm{o}}$ :

$B \sim 2.9 \times 10^{-5}\left( \pm 0.4 \times 10^{-5}\right) \sqrt{\rho_{\mathrm{o}}}$

with $\rho_{\mathrm{o}}\left(\mathrm{cm}^{-3}\right)$ and $B$ (Gauss). The accuracy on the angle and on the relation between $B$ and $\rho_{\mathrm{o}}$ is given by the uncertainty on the determination of the frequencies and on the measurement of the characteristic lengths. In Fig. 10, we plot the variation of the magnetic field strength $B$ versus the density $\rho_{\mathrm{o}}$. For a reasonable range of $B(10$ $70 \mathrm{G}$ ), the density in the slab ranges between $1.2 \times 10^{11}$ and $5.6 \times 10^{12} \mathrm{~cm}^{-3}$. These results are in agreement with Eq. (9): for a given density $\rho_{\mathrm{o}}$ the magnetic field strength is higher than $B_{0}\left(\rho_{\mathrm{o}}\right)$.

The results are not very sensible to the effective value of the filament temperature: for $5000 \mathrm{~K}<T_{\mathrm{o}}<15000 \mathrm{~K}$ the value of the angle $\phi$ and the coefficient in Eq. (15) change by less than $5 \%$. This is due to the fact that the Alfvén and fast magnetoacoustic modes are not very sensitive to the temperature for reasonable ranges of density and magnetic field strength in the filament (see Eqs. (12) and (13)). Only the slow magnetoacoustic modes are sensitive to the temperature (e.g. Joarder et al. 1997) but their frequencies are too low to be detected with our present observational constraints. Actually, the measurement of the primary frequency of the slow sausage mode should allow us to obtain a good estimation of the temperature in the filament.

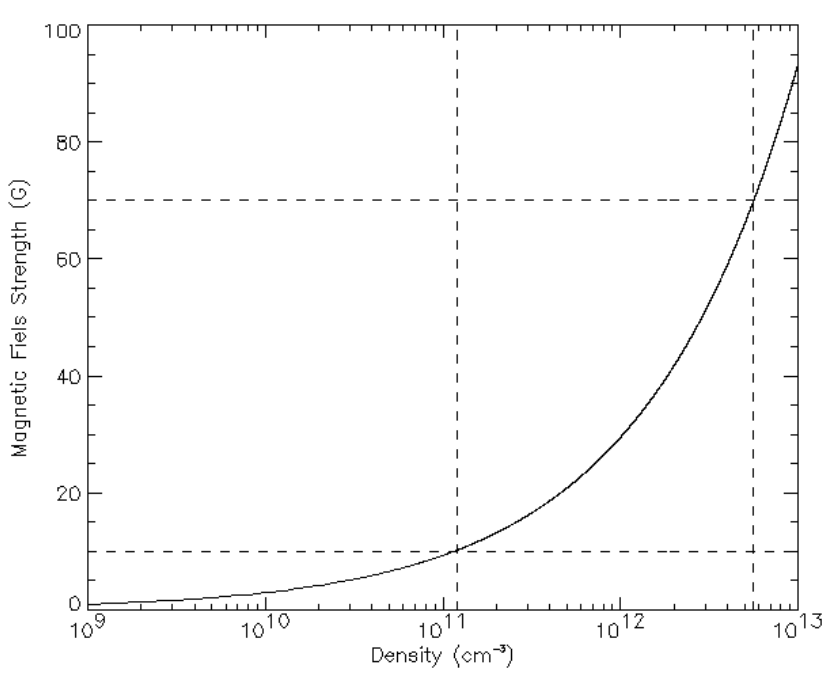

Fig. 10. Evolution of the magnetic field strength vs. the density in the slab. The reasonable range for $B$ is delimited by the horizontal dashed lines.

\section{Discussion and conclusions}

SUMER observations of active region filament oscillations have been obtained in the HeI line at $584.33 \AA$ with a total duration $\sim 7 \mathrm{~h} 30 \mathrm{~min}$ and with a small exposure time $\sim 30 \mathrm{~s}$. A Fourier analysis of the line-ofsight velocity time series allowed to evidence oscillations in the three ranges of periodicities defined by Molowny-Horas et al. (1997): short ( $<5 \mathrm{~min})$ periodicities for the quiet region, intermediate (5-20 min) and long (40$90 \mathrm{~min})$ periodicities for the filament.

Starting from a theoretical study of the modes of oscillations using the prominence model described by Joarder \& Roberts (1993), we compared the observed frequencies to the theoretical frequencies of Alfvén and magnetoacoustic waves. In this frame, we successfully identified the observed frequencies in terms of Alfvén and magnetoacoustic primary mode frequencies (see Table 5).

The knowledge of the frequencies provides in fact a diagnostic of the observed filament: determination of the angle $\phi \sim 18^{\circ}$, and of the value of the magnetic field strength versus the density $\rho_{\mathrm{o}}$ in the slab. The results are in agreement with typical values reported by Leroy et al. (1984). To complete the diagnostic, we should have to determine the density and the temperature in the filament or in its environment using diagnostic lines ratios. Since in this model $\rho_{\mathrm{o}} T_{\mathrm{o}}=\rho_{\mathrm{e}} T_{\mathrm{e}}$, we would obtain the density in the slab and therefore the magnetic field strength (Eq. (15)).

In order to improve and develop the method of diagnostic that we have discussed in this paper, new observations of active region filament using SoHO (SUMER, CDS) in coordination with THEMIS (Tenerife) observations are under consideration: using high temporal resolution and long time observations, we study the oscillations in active region filaments and in quiescent filaments with different values of $l$ and $a$, and with an observational diagnostic of the density and the temperature in the slab 
in order to estimate the magnetic field strength in the filament. Finally from a theoretical point of view, some efforts should be made in order to develop a more realistic model of prominence (e. g. to take into account the gravity, the geometry of the magnetic field and the fine structure). Although from a comparison of the theory and of the observations, we have obtained reasonable values of the physical parameters, one should verify the sensitivity of the results to a specific model. The identification of the mode frequencies should be made easier with a better estimation of the dimensionless parameter $\left(\frac{a}{l}\right)$. For the width $a$, this should be obtained by using high resolution $\mathrm{H} \alpha$ images and for the length $l$ by a more accurate determination of the photospheric sites of the magnetic field lines supporting the filament.

Acknowledgements. The authors thank P. Lemaire for discussion on the SUMER characteristics, and the referees for fruitful comments. SUMER is financially supported by DLR, CNES, NASA and the ESA PRODEX program (Swiss contribution). SoHO is a mission of international cooperation between ESA and NASA.

\section{References}

Balthasar, H., Knölker, M., Wiehr, E., \& Stellmacher, G. 1986, A\&A, 163, 343

Balthasar, H., Stellmacher, G., \& Wiehr, E. 1988, A\&A, 204, 286

Bashkirtsev, V. S., Kobanov, N. I., \& Mashnich, G. P. 1983, Sol. Phys., 82, 443

Bashkirtsev, V. S., \& Mashnich, G. P. 1984, Sol. Phys., 91, 93

Blanco, S., Bocchialini, K., Costa, A., et al. 1999, Sol. Phys., 186, 281

Brault, J. W., \& White, O. R. 1971, A\&A, 13, 169

Bocchialini, K., Costa, A., Domenech, G., et al. 2001, Sol. Phys., to be published

Cushman, G. W., \& Rense, W. A. 1978, Sol. Phys., 58, 299

Delaboudinière, J. P., \& Crifo, J. F. 1976, Space Research XVI, Akademie-Verlag Berlin, 803

Dupree, A. K., \& Reeves, E. M. 1971, ApJ, 165, 599

Dupree, A. K., Huher, M. C. E., Noyes, R. W., et al. 1973, ApJ, 182, 321

Galindo-Trejo, J. 1987, Sol. Phys., 108, 265

Heasley, J. N., Mihalas, D., \& Poland, A. I. 1974, ApJ, 192, 181
Joarder, P. S., \& Roberts, B. 1992a, A\&A, 256, 264

Joarder, P. S., \& Roberts, B. 1992b, A\&A, 261, 625

Joarder, P. S., \& Roberts, B. 1993, A\&A, 277, 225

Joarder, P. S., Nakariakov, V. M., \& Roberts, B. 1997, Sol. Phys., 173, 81

Kippenhahn, R., \& Schlüter, A. 1957, Z. Astrophys., 43, 36

Labrosse, N., Gouttebroze, P., \& Vial, J. C. 1999, 9th European Meeting on Solar Physics, ESA SP-448, 503

Lemaire, P. 2000, private communication

Leroy, J. L., Bommier, V., \& Sahal-Brechot, S. 1984, A\&A, 131,33

Malherbe, J. M., Schmieder, B., \& Mein, P. 1981, A\&A, 102, 124

Malherbe, J. M., Schmieder, B., Mein, P., \& TandbergHanssen, E. 1987, A\&A, 172, 316

Molowny-Horas, R., Oliver, R., Ballester, J. L., \& Baudin, F. 1997, Sol. Phys., 172, 181

Molowny-Horas, R., Oliver, R., Ballester, J. L., \& Baudin, F. 1998, New Perspectives on Solar Prominences, ed. D. Webb, D. Rust, \& B. Schmieder, IAU Colloq. 167

Oliver, R., Ballester, J. L., Hood, A. W., \& Priest, E. R. 1992, ApJ, 400, 369

Oliver, R. 1999, 9th European Meeting on Solar Physics, ESASP, 448, 425

Priest, E. R. 1982, Solar magnetohydrodynamics (Reidel, Dordrecht), 91

Régnier, S., Solomon, J., Vial, J. C., Amari, T., \& Mickey, D. 1999, 9th European Meeting on Solar Physics, ESA-SP 448, 519

Régnier, S., Solomon, J., \& Vial, J. C. 2001, Recent Insights into the Physics of the Sun and Heliosphere Highlights from SOHO and Other Space Missions, ASP Conf. Ser. 200, ed. P. Brekke, B. Fleck, \& J. B. Gurman, submitted

Roberts, B. 1992, in Proc. Cowling Memorial Meeting, Geophys. Astrophys. Fluid Dyn., 62, 83

Schmieder, B., Malherbe, J. M., Poland, A. I., \& Simon, G. 1985, A\&A, 153, 64

Schmieder, B. 1989, in Dynamics and Structure of quiescent solar prominences, ed. E. Priest (Kluwer, London), 15

Thompson, W. T., \& Schmieder, B. 1991, A\&A, 243, 501

Vial, J. C. 1998, New Perspectives on Solar Prominences, ed. D. Webb, D. Rust, \& B. Schmieder, IAU Colloq. 167

Vial, J. C. 2000, unpublished

Wiehr, E., Stellmacher, G., \& Balthasar, H. 1984, Sol. Phys., 94,285

Wilhelm, K., et al. 1995, Sol. Phys., 162, 189

Yi, Z., Engvold, O., \& Keil, S. L. 1991, Sol. Phys., 132, 63 